\title{
Dynamic space charge behaviour in polymeric DC cables
}

\section{Rasmussen, Claus Nygaard; Holbøll, Joachim; Henriksen, Mogens}

\section{Published in:}

Proceedings of the 11. International Symposium on Electrets

Link to article, DOI:

10.1109/ISE.2002.1042936

Publication date:

2002

Link back to DTU Orbit

Citation (APA):

Rasmussen, C. N., Holbøll, J., \& Henriksen, M. (2002). Dynamic space charge behaviour in polymeric DC cables. In Proceedings of the 11. International Symposium on Electrets (pp. 23-26). IEEE. https://doi.org/10.1109/ISE.2002.1042936

\section{General rights}

Copyright and moral rights for the publications made accessible in the public portal are retained by the authors and/or other copyright owners and it is a condition of accessing publications that users recognise and abide by the legal requirements associated with these rights.

- Users may download and print one copy of any publication from the public portal for the purpose of private study or research.

- You may not further distribute the material or use it for any profit-making activity or commercial gain

- You may freely distribute the URL identifying the publication in the public portal

If you believe that this document breaches copyright please contact us providing details, and we will remove access to the work immediately and investigate your claim. 


\title{
Dynamic space charge behaviour in polymeric DC cables
}

\author{
C.N. Rasmussen, J.T. Holbøll \& M. Henriksen, \\ Institute of Electric Power Engineering, Technical University of Denmark
}

\begin{abstract}
The use of extruded insulation for DC cables involves a risk of local electric field enhancement, caused by a space charge build-up within the dielectric. In this work, the theory of charge generation and transport in polymers is applied in a numerical computer model in order to predict the formation and transport of space charges in a polymeric dielectric. The model incorporates the processes of field assisted electron-hole pair generation from impurity atoms, trapping and charge injection at the electrodes. Its aim has been to study the field- and temperature dependent dynamic behaviour of a dielectric. Results obtained using this model-based framework are compared to measurement results obtained from Laser Induced Pressure Pulse (LIPP) space charge measurements as well as conductivity measurements on selected cable type samples.
\end{abstract}

\section{Introduction}

Since the build-up of space charge determines the electric field distribution in a dielectric subjected to a constant field it is important to be able to explain the processes involved in such a charge build-up. In the case of a High Voltage Direct Current (HVDC) electric power cable, the aim is to prevent local field enhancement by avoiding the build-up of space charge to the largest possible extend.

A large research effort has been invested in trying to find the combination of materials, most suitable for an HVDC-cable insulation system. However some discrepancy still remains between results obtained from measurement of space charge and conductivity in polymer samples, and the deductions made possible from available theory. This work attempts to explain typical properties of measurement results by using a model founded on established theory. Our ambition is to try and give answers to the following set of questions: Firstly, can the decaying current that is observed when a voltage is applied to a test sample, be explained by using the basic equations, combined with a set of simple assumptions about the insulation system? Secondly, can the experimentally observed transient space charge phenomenon be demonstrated to proceed in accordance with the results generated by our model?

\section{Theoretical background}

If the magnitude of an electric field is changed from one constant value to another, the insulation system will move from one steady state situation to that of another. During this transition period, the electric charge distribution in the bulk of the insulation material and the surface charge density on the electrodes will change. The new charge distribution is determined by the charge mobility, density of free carriers and permittivity in the bulk of the sample, as well as the injection (and extraction) currents at the electrodes. There are two basic equations, which must be combined in order to describe the electrical behaviour of the sample. Those equations are a) Maxwell's third equation, which specifies the relationship between charge density and electric flux density and b) the continuity equation, stating that the accumulation of charge is related to the spatial variations in current density. At any given time the current density in the system will be determined by (1).

$$
J=\sum_{i=1}^{i_{\max }}\left(\rho_{i} \cdot \mu_{i} \cdot E+D_{0 i} \cdot \nabla \rho_{i}\right)+\frac{\partial D}{\partial t}
$$

Equation (1) states that the current can be divided into a transport current driven by the electric field, a diffusion current driven by the gradient in charge density and a displacement current. The different types of charge carriers make up the transport- and diffusion currents. These carriers can be electrons or ions. In some cases it is can be an advantage to include the concept of electron-hole transport but it is also possible to regard a moving electron-hole as an electron jumping between positive donor-sites. The displacement current can be disregarded because of the large time-scales involved. The diffusion current is normally very small, however, it should be taken into account in places where spatial gradients in charge density are very large. 
Equation (1) does not apply at the interfaces. This is due to the fact that electrons have to jump a barrier in order to move from the electrode to the bulk of the insulation. This barrier is due to the difference in free-electron energy between the insulator and the electrode, which depends on the materials involved. The theory of charge injection involves several mechanisms. Here we assume that injection can be described by the Schottky mechanism [1], which results in a current given by (2).

$$
J_{s}=\frac{4 \cdot \pi \cdot e \cdot m_{e} \cdot k_{b}{ }^{2} \cdot T^{2}}{h^{3}} \cdot \exp \left[\frac{-\Phi}{k_{b} \cdot T}\right] \cdot \exp \left[\frac{e}{2 \cdot k_{b} \cdot T} \cdot \sqrt{\frac{e \cdot|E|}{\pi \cdot \varepsilon}}\right]
$$

In (2) $\Phi[\mathrm{eV}]$ is the potential barrier height. If the injected carriers are electrons, then (2) will give the current density at the cathode, while the anode current density is still given by (1) since free electrons are able to move freely down the Schottky barrier from insulator to electrode.

\section{Model description}

A numerical model, using the basic equations, has been formulated in MATLAB. The model uses a finite difference method to make a dynamic simulation of a radial geometry, i.e. the model is 2dimentional with radius and time as dimensions. The 2-dimentional "space" is divided into a number of radial steps and time steps. After setting the initial conditions, the program runs in a loop through the time steps. In each time step the charge density is assumed constant and the electric field, carrier mobility, free carrier density and current density across the insulation system can be calculated. The current density is then used to calculate the charge density in the next time step. In addition to the basic equations some assumptions have been made. Electrons are assumed to the only movable charge carriers and they are injected at the cathode. At the same time electrons are extracted from a number of donors present in the material at a density of $\mathrm{N}_{\mathrm{D}}$. When an electron is extracted from a donor, a positive hole is left behind. The holes are assumed to be immovable. The density of free electrons extracted from donors is calculated using (3).

$$
n_{e_{-} \text {donor }}=n_{\text {hole }}=N_{D} \cdot \exp \left[\frac{-E_{D}}{k_{b} \cdot T}\right] \cdot \exp \left[\frac{e}{2 \cdot k_{b} \cdot T} \cdot \sqrt{\frac{e \cdot|E|}{\pi \cdot \varepsilon}}\right]
$$

In (3) $E_{D}[e V]$ is the energy gab between the donors and the conduction band. The mobility of electrons is calculated by assuming trap-limited mobility [1], [2]. The electrons are assumed to have an effective mobility (4), where $\theta$ is the fraction of electrons (5) which at any given time are free to move while the rest are trapped.

$$
\begin{aligned}
& \mu_{e}=\frac{e \cdot a}{\sqrt{3 \cdot k_{b} \cdot m_{e} \cdot T}} \cdot \theta \\
& \theta=\frac{N_{c}}{N_{A}} \cdot \exp \left[\frac{-E_{A}}{k_{b} \cdot T}\right] \cdot \exp \left[\frac{e}{2 \cdot k_{b} \cdot T} \cdot \sqrt{\frac{e \cdot|E|}{\pi \cdot \varepsilon}}\right]
\end{aligned}
$$

$\mathrm{N}_{\mathrm{c}} \sim 10^{29} \cdot \mathrm{m}^{3}$ is the effective density of states in the conduction band; $\mathrm{E}_{\mathrm{A}}$ is the energy gab between the acceptors (traps) and the conduction band and $a$ is the effective distance between electron collisions. Extraction from donors and acceptors is assumed to be subject to the Poole-Frenkel effect.

\section{Results and discussion}

Simulations have been carried out using values resulting in conductivities in the range $\sigma \in\left[10^{-16} ; 10^{-12}\right]$ $\mathrm{S} / \mathrm{m}$. Different combinations of interface barrier height; donor concentration and bulk mobility have been simulated. The results can basically be divided in two: Firstly, if the initial cathode injection current is larger than the initial transport current in the bulk then the injection current will fall until an 
equilibrium has been established. This occurs when the injected charge has formed a barrier that limits the current. In this case a steady state situation with negative charge inside the sample will arise. If the electrode barrier is very low the end result will be the space charge limited (SCL) case [1]-[3]. The negative charge reduces the current so that the steady-state current is much smaller than the initial current. This leads to the assumption that the build-up of injected charge can explain the observed time dependent current density in a polymer sample after the application of voltage. The current is observed to decay and can be fitted well using the expression $I \propto I_{s} \cdot\left[1+(t / \tau)^{-n}\right], n \in 0.7-1$. Figure 1 shows a measured current density as well as the simulated current density at the cathode.

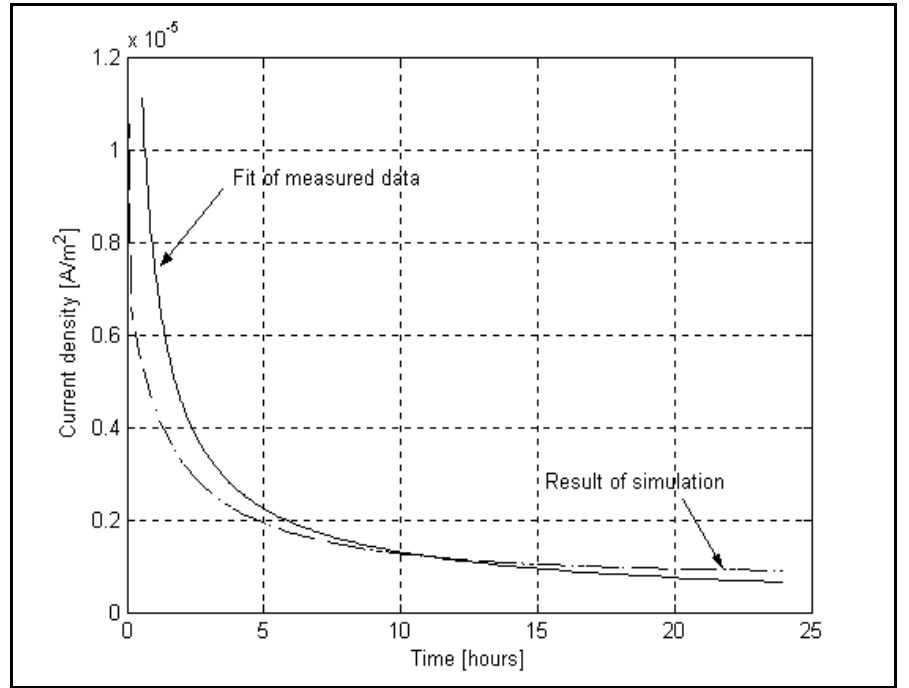

Figure 1: Fit of a typical J vs. t measurement, on a cable sample. The dotted line is the simulated current vs. time profile with $\Phi=1.3$ $\mathrm{eV}, \mathrm{E}_{\mathrm{D}}=0.4 \mathrm{eV}$ and $\mathrm{E}_{\mathrm{A}}=1.05 \mathrm{eV}$.

The simulated current is similar to the measured one. Varying the bulk conductivity varies $\tau$ and the height of the injection barrier will determine the steady-state current $\left(I_{s}\right)$. If the injection barrier is increased so that the initial $\mathrm{I}_{\mathrm{s}}<\mathrm{I}_{\text {bulk }}$ then a positive charge will form next to the cathode. This positive charge will grow and increase the field until the injection current matches the bulk current and a steady state develops. This situation is show in figure 2 .

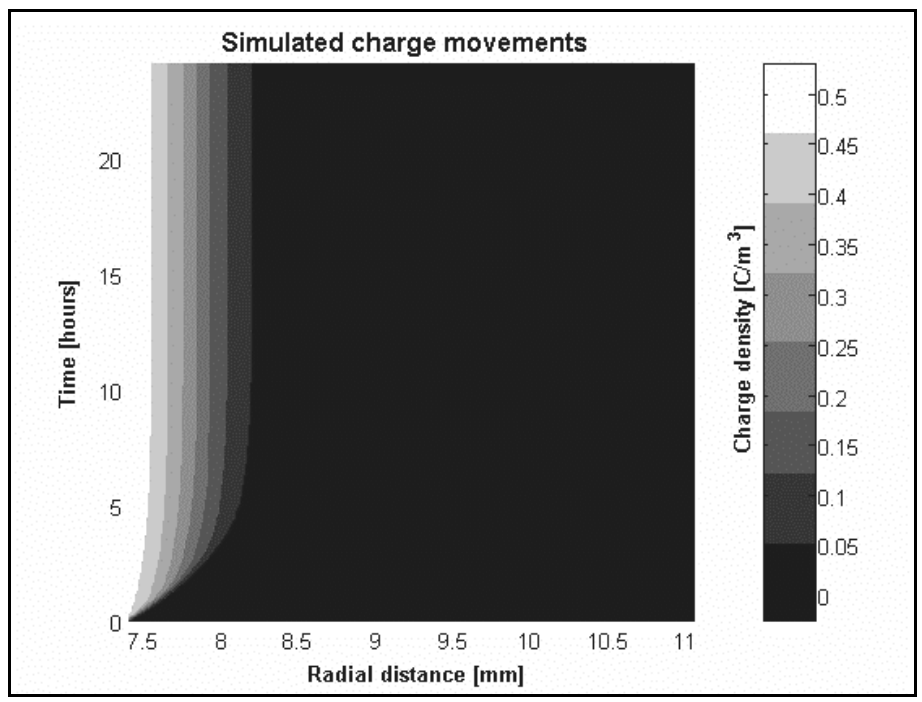

Figure 2: Simulated charge density in a sample with initial $\mathrm{I}_{\mathrm{s}}<\mathrm{I}_{\text {bulk }}$. $\sigma \approx 5 \cdot 10^{-16} \mathrm{~S} / \mathrm{m}$. With $\Phi=1.45 \mathrm{eV}, \mathrm{E}_{\mathrm{D}}=0.4 \mathrm{eV}$ and $\mathrm{E}_{\mathrm{A}}=1.05 \mathrm{eV}$. 
Figure 2 is a contour plot showing the result of a 24-hour simulation of the space charge development inside a cable. The inner and outer diameters are 7.35 and $11.05 \mathrm{~mm}$ respectively. The voltage applied to the inner conductor is $-60 \mathrm{kV}$. The plot does not show the electrode charges because the charge density, in principle, reaches an infinite value on the electrodes. Figure 3 shows a measured charge density, which should be compared to the simulation from figure 2. Positive charge clearly forms near the cathode and this situation corresponds to an initially low injection current and the steady state situation is not far from what is shown in figure 2 .

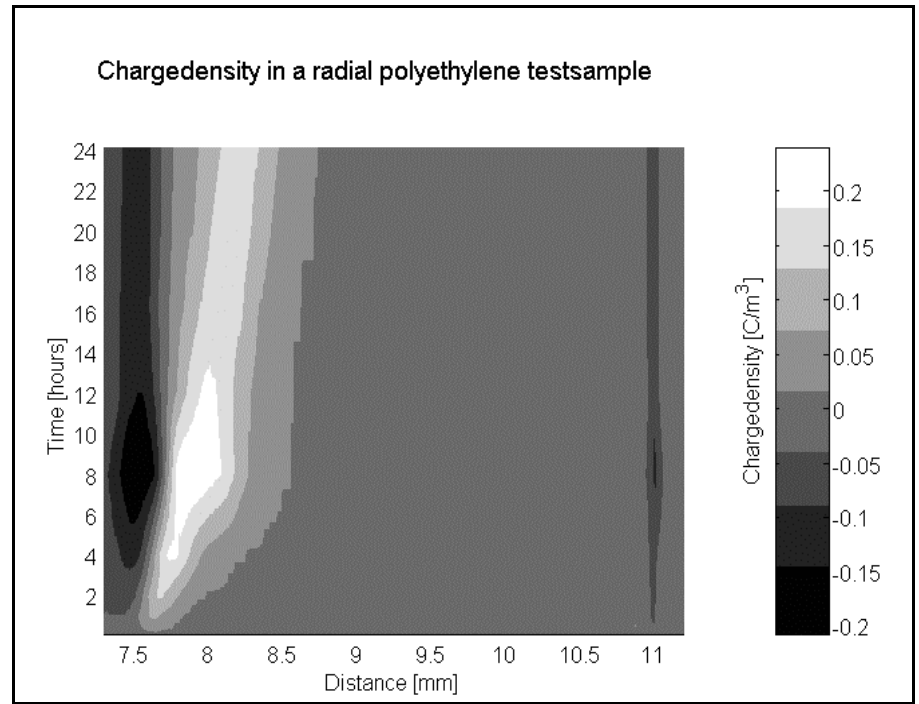

Figure 3: Measured charge movement in an LDPE test cable. With voltage removed. The positive charge inside the sample induces negative charge on the electrodes at $\mathrm{r} \approx 7.5 \mathrm{~mm}$ and $\mathrm{r} \approx 11 \mathrm{~mm}$.

\section{Conclusions}

The declining current and positive charge build-up close to the cathode has been modelled by assuming one free charge carrier, injection at the cathode and the presence of electron donors in the bulk. The obvious similarity between our simulation results and measured results points towards a fairly accurate model formulation. The model does not take into account the formation and transport of ions, nor does it incorporate the orientation of dipoles in the field. Omitting these factors does however, not seem to have prevented us from deriving at a useful model. A further possible shortcoming in our model is that it takes for granted the Poole-Frenkel effect, as being the mechanism of field-enhanced conductivity; an assumption that may not be accurate. The model can be used to put limits to the densities and energy levels of traps and donors in the polymer. Only a very limited range of values will produce simulation results that are similar to what can be obtained from measurements. The current vs. time and space charge development in a polymer is determined both by bulk mobility and injection properties, which demonstrates that the electrode material to a large extend will decide the apparent conductivity of the sample.

\section{Acknowledgements}

The authors would like to convey gratitude to Jesper Hjerrild for his work on the test set-up, and to Erik Østergaard Madsen for his helpful contribution to the design of the numerical computer model.

\section{References}

[1] - Engineering dielectrics, Volume IIA. Electrical Properties of Solid Insulating Materials: Molecular Structure and Electrical Behaviour. R. Bartnikas \& R.M. Eichhorn, ASTM 1983.

[2] - Electrical Degradation and Breakdown in Polymers. L. A. Dissado and J. C. Fothergill, Peter Peregrinus Ltd, London, 1992.

[3] - Physics of Dielectrics. R. Coelho, Elsevier Scientific Publishing Company, 1979. 